\title{
Asymptotic Completeness for Four-Body Schrodinger Operators with Short-Range Interactions
}

By

\author{
Hideo TAMURA*
}

\section{$\S$ 0. Introduction}

The present work is a continuation to the previous one [15] in which the author has studied the problem of asymptotic completeness in the case of threebody systems with short-range pair interactions with a view to making transparent the proof of asymptotic completeness in the remarkable work by SigalSoffer [13] which deals with the case of general $N$-body systems. The proof in [15] has been in principle based on the same idea as in [13] but several new ingredients have been added to the techniques developed there. In particular, the proof does not have required a phase space partition of unity with the property that the boundaries of its support lie in the classically forbidden region. The construction of such a phase space partition of unity is one of the most essential steps in the original proof by [13]. The aim of this work is to develope further the argument used in [15] to prove the asymptotic completeness for four-body systems with short-range pair interactions. The author hopes that the previous and present works reveal the difficulties to be overcome in the future study towards proving the asymptotic completeness for general $\mathrm{N}$ body, $N \geqq 5$, systems.

The precise formulation of the obtained result requires several complicated but basic notations and definitions in many-body scattering theory.

For notational brevity, we here consider only a simple system of four particles with identical masses normalized by $m_{j}=1,1 \leqq j \leqq 4$, moving in the three-dimensional space $\boldsymbol{R}^{3}$. For such a system, the configuration space $X$ in the center of mass frame is given by

$$
X=\left\{r=\left(r_{1}, r_{2}, r_{3}, r_{4}\right) \in \boldsymbol{R}^{3 \times 4}: \sum_{j=1}^{4} r_{j}=0\right\}
$$

with the usual scalar product

$$
r \cdot \tilde{r}=\sum_{j=1}^{4} r_{j} \cdot \tilde{r}_{j}
$$

Communicated by S. Matsuura, November 9, 1989.

1991 Mathematics Subject Classification : 35P

* Department of Mathematics, Ibaraki University, Mito, Ibaraki 310, Japan 
and also the energy Hamiltonian $H$ (Schrödinger operator) takes the form

$$
H=-\frac{1}{2} \Delta+V \quad \text { on } \quad L^{2}(X),
$$

where $\Delta$ denotes the Laplacian on $X$ and the interaction $V(r)$ is given by a sum of pair potentials;

$$
V(r)=\sum_{1 \leqq j<k \leqq 4} V_{j k}\left(r_{j}-r_{k}\right) .
$$

The real-valued pair potential $V_{j k}(y), y \in \boldsymbol{R}^{3}$, is assumed to have the following decaying property:

$$
\left|V_{j k}(y)\right| \leqq C(1+|y|)^{-\rho} \quad \text { for some } \rho>1 \text {. }
$$

We may assume that $1<\rho<2$. Throughout the entire discussion, the constant $\rho$ is used with the meaning ascribed above and also assumption (V) is always assumed to be satisfied. Under this assumption, the operator $H$ formally defined by (0.2) admits a unique self-adjoint realization in $L^{2}(X)$. We denote it by the same notation $H$.

Let $a=\left\{C_{1}, \cdots, C_{l}\right\}, 2 \leqq l \leqq 4$, be a cluster decomposition of the set $\{1,2,3,4\}$ into non-empty disjoint subsets. For pair $(j, k), 1 \leqq j<k \leqq 4$, we write $j a k$ if $j$ and $k$ are in the same cluster and $\sim j a k$ if they are in different clusters. For given cluster decomposition $a$, we further define the configuration space $Y_{a}$ of the internal motion within the clusters $C_{k}$ in $a$ by

$$
Y_{a}=\left\{r \in X: \sum_{j \in C_{k}} r_{j}=0 \text { for } C_{k} \in a\right\}
$$

and the configuration space $Z_{a}$ of the relative motion of the clusters in $a$ by

$$
Z_{a}=\left\{r \in X: r_{j}=r_{k} \text { for pair }(j, k) \text { with } j a k\right\} \text {. }
$$

We denote by $\#(a)$ the number of clusters in $a$. The spaces $Y_{a}$ and $Z_{a}$ are identified with the $\mu_{a}$-dimensional and the $\nu_{a}$-dimensional Euclidean spaces with $\mu_{a}=3 \times(4-\#(a))$ and $\nu_{a}=3 \times(\#(a)-1)$, respectively, and also the space $X$ is decomposed as the orthogonal sum of spaces $Y_{a}$ and $Z_{a}$ with respect to the scalar product $(0.1) ; X=Y_{a} \oplus Z_{a}$.

Let $a$ be again a cluster decomposition with $2 \leqq \#(a) \leqq 4$. Then we define the truncated Hamiltonian $H_{a}$ by

$$
H_{a}=K_{a} \otimes I d+I d \otimes T_{a} \quad \text { on } \quad L^{2}(X)=L^{2}\left(Y_{a}\right) \otimes L^{2}\left(Z_{a}\right),
$$

Id being the identity operator, where the Hamiltonian $K_{a}$ acting on $L^{2}\left(Y_{a}\right)$ is defined by

$$
K_{a}=-\frac{1}{2} \Delta+\sum_{j a k} V_{j k}\left(r_{j}-r_{k}\right)
$$

and $T_{a}=-(1 / 2) \Delta$ acting on $L^{2}\left(Z_{a}\right)$ is the kinetic energy operator of the center of mass motion of the clusters in $a$. If $\#(a)=4$, then we define $Y_{a}$ as $Y_{a}=C$ 
(scalar field) and $K_{a}$ as zero, so that $T_{a}=H_{0}=-(1 / 2) \Delta$ becomes the free Hamiltonian acting on $L^{2}\left(Z_{a}\right)=L^{2}(X)$.

We further introduce notations. Let $m_{a}, 0 \leqq m_{a} \leqq+\infty$, be the number of bound state energies of $K_{a}$ considered on the space $L^{2}\left(Y_{a}\right)$ with repetition according to the multiplicities. Such a bound state energy is called a threshold energy of the Hamiltonian $H$. Under assumption (V), we know ([7]) that $K_{a}$ has no positive bound state energies and that the set of all threshold and bound state energies of $H$ is closed and countable. A pair $\alpha=(a, m), 1 \leqq m \leqq m_{a}$, is called a channel. With the channel $\alpha$, we associate the following notions: (0) $\varepsilon_{\alpha} \leqq 0$, bound state energy of $K_{a}$; (i) $\phi_{a} \in L^{2}\left(Y_{a}\right)$, normalized eigenstate corresponding to $\varepsilon_{a}$; (ii) $H_{\alpha}=\varepsilon_{\alpha}+T_{a}$, channel Hamiltonian on $L^{2}\left(Z_{a}\right)$; (iii) $J_{\alpha}: L^{2}\left(Z_{a}\right) \rightarrow L^{2}(X)$; $J_{a} u=\phi_{\alpha} \otimes u$, channel identification operator ; (iv) $\Omega_{\alpha}^{ \pm}: L^{2}\left(Z_{a}\right) \rightarrow L^{2}(X)$, channel wave operator, defined by

$$
\Omega_{\alpha}^{ \pm}=\mathrm{s}_{t \rightarrow \pm \infty}-\lim _{\infty} \exp (i t H) J_{\alpha} \exp \left(-i t H_{\alpha}\right) .
$$

Under assumption (V), we know ([12]) that the channel wave operators $\Omega_{\alpha}^{ \pm}$ exist and that their ranges are orthogonal to each other. If $\psi$ is in Range $\Omega_{\alpha}^{ \pm}$, then by definition it follows that there exists $\phi_{\alpha}^{ \pm} \in L^{2}\left(Z_{a}\right)$ such that the state $\exp (-i t H) \psi$ behaves like

$$
\exp (-i t H) \psi \sim \exp \left(-i l \varepsilon_{a}\right) \phi_{a} \otimes \exp \left(-i t H_{a}\right) \phi_{a}^{ \pm} \quad \text { as } \quad t \rightarrow \pm \infty .
$$

Let $P_{I I}: L^{2}(X) \rightarrow L^{2}(X)$ be the eigenprojection of $H$ associated with point spectrum. Roughly speaking, the problem of asymptotic completeness is to study the asymptotic behavior as $t \rightarrow \pm \infty$ of the scattering state $\exp (-i t H) \psi$ with $\phi \in$ Range $\left(I d-P_{I I}\right)$. The following main theorem says that such a scattering state behaves like superpositions of the states as in (0.6) as $t \rightarrow \pm \infty$.

Theorem (asymptotic completeness). Let the notations be as above. Assume $(V)$. Then one has

$$
\text { Range }\left(I d-P_{H}\right)=\oplus_{\alpha} \text { Range } \Omega_{\alpha}^{ \pm} \text {. }
$$

Besides the work [13], there are many works dealing with the problem of asymptotic completeness for many-body systems. An extensive list of related references can be found in [2] and [13]. In the case of three-body systems, the most general result has been obtained by Enss [5], including the case of long-range interactions. However, there does not seem to be so many works dealing with the case of $N$-body, $N \geqq 4$, systems. For example, Hagedorn [8] has proved the asymptotic completeness for four-body systems with a certain class of pair potentials falling off faster than $|y|^{-\nu}, \nu>2$, by use of the Faddeev equation method. We also note that in the recent work [10], Kitada uses the argument based on the work by Enss [6] to prove the asymptotic completeness for general $N$-body systems under an additional assumption that all subsystem 
Hamiltonians have only a finite number of bound state energies.

\section{$\S 1$. Reduction to the Main Lemma}

We here explain the strategy of proof briefly. The proof is based on the same idea as in the previous work [15] and is done by reducing it to the proof of the main lemma below (Lemma 1.1).

We first fix an energy $E$ arbitrarily. Assume that $E$ is neither a threshold energy nor a bound state energy of $H$. Since the set of such threshold and bound state energies is closed and countable, we can take a small open interval $\Gamma$ around $E$ avoiding the threshold and bound state energies of $H$. Let $g_{0} \in$ $C_{0}^{\infty}(\Gamma)$ be a non-negative smooth function with support in $\Gamma$ such that $g_{0}=1$ in a small neighborhood of $E$.

We now follow the standard argument as in Section 2 of [13]. Assume the asymptotic completeness for two- and three-subsystem Hamiltonians. Let $\psi \in$ Range $\left(I d-P_{H}\right)$. Then the main theorem follows, if it can be proved that for any $\varepsilon>0$ small enough, there exist $\phi_{a, \varepsilon}^{ \pm} \in L^{2}(X)$ such that

$$
\lim _{t \rightarrow \pm \infty}\left\|\exp (-i t H) g_{0}(H) \psi-\sum_{\#(a)=2} \exp \left(-i t H_{a}\right) \phi_{a, \varepsilon}^{ \pm}\right\|_{X}<\varepsilon,
$$

where $\|\cdot\|_{X}$ denotes the $L^{2}$ norm in $L^{2}(X)$ and the summation $\sum_{\#(a)=2}$ is taken over all two-cluster decompositions $a$. We shall prove this for the case $E>0$ and $t \rightarrow \infty$ only. The other cases can be dealt with in a similar way.

1.1. To prove (1.1), we first introduce a partition of unity on $X$. We fix a coordinate system on $X$ arbitrarily and write it as $x=\left(x_{1}, x_{2}, x_{3}\right)$ with $x_{j} \in \boldsymbol{R}^{3}$, $1 \leqq j \leqq 3$. Let $S$ be the unit sphere in $X$. Let $a$ be a two-cluster decomposition. Then we define the closed subset $S_{a}$ in $S$ by

$$
S_{a}=\left\{\omega=\left(\omega_{1}, \omega_{2}, \omega_{3}, \omega_{4}\right) \in S: \omega_{j}=\omega_{k} \text { for }(j, k) \text { with } j a k\right\} .
$$

By definition, it follows immediately that the sets $S_{a}$ and $S_{b}, a \neq b$, with $\#(a)=$ $\#(b)=2$ do not intersect each other.

We now introduce a non-negative smooth partition of unity on $X$, $\left\{j_{a}(x)\right\}_{\#(a)=2}$, with the following properties: $(j .0) \sum_{\#(a)=2} j_{a}(x)=1$ on $X ;(j .1)$ For $|x|>1, j_{a}(x)$ has support in a conical neighborhood of $S_{a}$ (and hence the gradient $\nabla_{x} j_{a}$ vanishes in a small conical neighborhood of $\left.S_{a}\right) ;(j .2)$ For any multi-index $\alpha,\left|\partial_{x}^{\alpha} j_{a}(x)\right| \leqq C_{\alpha}(1+|x|)^{-|\alpha|}$;

$$
j_{a}(x) \text { vanishes on } Y_{a}
$$

for $|x|>1, Y_{a}$ being defined by (0.3). It is not difficult to see that such a partition of unity really exists.

1.2. Let $g_{0} \in C_{0}^{\infty}(\Gamma)$ be as above and let $g_{1} \in C_{0}^{\infty}(\Gamma)$ be also a non-negative 
function such that $g_{1}=1$ on the support of $g_{0}$, so that $g_{1} g_{0}=g_{0}$. By property ( $j .1), j_{a}\left(g_{1}(H)-g_{1}\left(H_{a}\right)\right): L^{2}(X) \rightarrow L^{2}(X)$ is compact and, by property $(j .2)$, the commutator $\left[g_{1}\left(H_{a}\right), j_{a}\right]$ is also compact. The state $\exp (-i t H) g_{0}(H) \psi$ is weakly convergent to zero as $t \rightarrow \infty$ and hence

$$
\lim _{t \rightarrow \infty}\left\|\exp (-i t H) g_{0}(H) \phi-\sum_{\#(a)=2} \exp \left(-i t H_{a}\right) \psi_{a}(t)\right\|_{X}=0,
$$

where

$$
\psi_{a}=g_{1}\left(H_{a}\right) \exp \left(i t H_{a}\right) j_{a} \exp (-i t H) g_{0}(H) \psi .
$$

The above term $\psi_{a}(t)$ can be rewritten in the integral form

where

$$
\phi_{a}(t)=g_{1}\left(H_{a}\right) j_{a} g_{0}(H) \phi+i \phi_{0 a}(t)+i \phi_{a}(t),
$$

$$
\dot{\phi}_{0 a}=\int_{0}^{t} g_{1}\left(H_{a}\right) \exp \left(i s H_{a}\right) j_{a}\left(H_{a}-H\right) \exp (-i s H) g_{0}(H) \phi d s
$$

and

$$
\phi_{a}=\int_{0}^{t} g_{1}\left(H_{a}\right) \exp \left(i s H_{a}\right)\left[H_{0}, j_{a}\right] \exp (-i s H) g_{0}(H) \psi d s,
$$

$H_{0}=-(1 / 2) \Delta$ being the free Hamiltonian on $X$.

1.3. To analyze the term $\phi_{a}(t)$, we further introduce a non-negative smooth partition of unity on $\boldsymbol{R}^{1},\left\{f_{\jmath}\right\}_{j=1}^{4}$, with the following properties : $(f .0) \sum_{j=1}^{4} f_{j}^{2}=1$ on $\boldsymbol{R}^{1}$;

(f.1) $\quad \operatorname{supp} f_{1} \subset(-\infty,-\sqrt{2 E} / 3), \quad f_{1}=1$ on $(-\infty,-\sqrt{2 E} / 2]$;

$$
\begin{aligned}
& \text { supp } f_{2} \subset(-\sqrt{2 E} / 2, \sqrt{2 E}(1-\kappa)), \quad f_{2}=1 \quad \text { on }[-\sqrt{2 E} / 3, \sqrt{2 E}(1-2 \kappa)] ; \\
& \operatorname{supp} f_{3} \subset(\sqrt{2 E}(1-2 \kappa), M), f_{3}=1 \text { on }[\sqrt{2 E}(1-\kappa), M / 2] ; \\
& \operatorname{supp} f_{4} \subset(M / 2, \infty), f_{4}=1 \text { on }[M, \infty),
\end{aligned}
$$

where $\kappa, 0<\kappa \ll 1$, and $M, M \gg 1$, are taken sufficiently small and large, respectively.

As in [13], we now define the operator $\gamma$ as

$$
\gamma=\frac{1}{2 i}\left\{(x /\langle x\rangle) \cdot \nabla_{x}+\nabla_{x} \cdot(x /\langle x\rangle)\right\}
$$

with

$$
\langle x\rangle=\left(1+|x|^{2}\right)^{1 / 2} .
$$

It is known (Theorem 3.2, [13]) that the operator $\gamma$ is self-adjoint in $L^{\prime}(X)$ with its natural domain $\mathscr{D}(\gamma)=\left\{u \in L^{2}(X): \gamma u \in L^{2}(X)\right\}$. According to property $(f .0)$, we decompose the term $\phi_{a}(t)$ under consideration into

where

$$
\phi_{a}(t)=\sum_{j=1}^{4} \phi_{j a}(t),
$$




$$
\phi_{j a}=\int_{0}^{t} g_{1}\left(H_{a}\right) \exp \left(i s H_{a}\right) f_{j}(\gamma)^{2}\left[H_{0}, j_{a}\right] \exp (-i s H) g_{0}(H) \psi d s \text {. }
$$

The following two lemmas imply (1.1) for the case $E>0$ and $t \rightarrow \infty$.

Lemma 1.0. Let $\phi_{j a}(t), 0 \leqq j \leqq 4$, be as above. Then one has the following statements :

(i) $\phi_{j a}(t), 0 \leqq j \leqq 2$, strongly converges in $L^{2}(X)$ as $t \rightarrow \infty$.

(ii) If $M$ is chosen large enough, then $\phi_{4 a}(t)$ also strongly converges as $t \rightarrow \infty$.

Lemma 1.1. For any $\varepsilon>0$ small enough, there exist $\phi_{a_{\bullet} \varepsilon} \in L^{2}(X)$ such that

$$
\limsup _{t \rightarrow \infty}\left\|\sum_{\#(a)=2} \exp \left(-i t H_{a}\right)\left(\phi_{3 a}(t)-\phi_{a, \varepsilon}\right)\right\|_{X}<\varepsilon \text { 。 }
$$

In the previous work [15], we have already proved Lemma 1.0 in the case of three-body systems. The arguments there apply without any essential changes also to the case of four-body systems in question. Thus the proof of main theorem is reduced to that of Lemma 1.1.

The remaining sections are devoted to proving Lemma 1.1. This lemma is proved through the study on the behavior as $t \rightarrow \infty$ of the outgoing state $f(\gamma) \exp (-i t H) g_{0}(H) \phi$ with $f \in C_{0}^{\infty}\left(\boldsymbol{R}^{1}\right)$ supported in $(0, \infty)$. The analysis for such an outgoing state occupies the most essential part of the proof of asymp. totic completeness in our approach.

\section{§2. Non-Propagation Estimate}

The most important result on which the proof of the main lemma is based is the non-propagation estimate in the classically forbidden region for the propagator $\exp (-i t H)$ which has been obtained by [3] and [13] for general $N$ body systems, including the case of long-range interactions.

2.1. The precise formulation of the result requires many notations.

We first introduce the following function space:

$$
S^{n}\left(R_{z}^{\nu}\right)=\left\{f \in C^{\infty}\left(R_{z}^{\nu}\right):\left|\partial_{z}^{\alpha} f\right| \leqq C_{\alpha}\langle z\rangle^{m-|a|}\right\} .
$$

Let $Y_{a}$ and $Z_{a}$ be defined by (0.3) and (0.4), respectively, and denote by $X^{\prime}$, $Y_{a}^{\prime}$ and $Z_{a}^{\prime}$ the spaces dual to $X, Y_{a}$ and $Z_{a}$, respectively. For given symbol $F \in S^{m}\left(Z_{a}^{\prime}\right)$, we define the pseudodifferential operator $F\left(D_{a}\right)$ by

$$
F\left(D_{a}\right) u=(2 \pi)^{-\nu / 2} \int \exp \left(i z_{a} \cdot q_{a}\right) F\left(q_{a}\right) \hat{u}\left(q_{a}\right) d q_{a},
$$

where $\nu$ is the space dimension of $Z_{a}$ and $\hat{u}\left(q_{a}\right)$ is the Fourier transform of $u$ :

$$
\hat{u}\left(q_{a}\right)=(2 \pi)^{-\nu / 2} \int \exp \left(-i z_{a} \cdot q_{a}\right) u\left(z_{a}\right) d z_{a}
$$


the integration with no domain attached being taken over the whole space. This abbreviation is often used throughout the discussion in the sequel.

We further introduce notations. For given cluster decompositions $a$ and $b$, we use the notation $b \subset a$ to indicate that $b$ is a refinement of $a$. Let the Hamiltonian $K_{a}$ acting on $L^{2}\left(Y_{a}\right)$ be as in (0.5). We know ([7]) that $K_{a}$ has no positive bound state energies. Denote by $\tilde{\Lambda}_{a}$ the set of all (non-positive) bound state energies of $K_{a}$. If $\#(a)=4$, then $\tilde{\Lambda}_{a}$ is defined as $\tilde{\Lambda}_{a}=\{0\}$. We further define the set $\Lambda_{a}$ by $\Lambda_{a}=\cup_{b \subset a} \tilde{\Lambda}_{b}$ and $\Sigma_{a}(E)$ by

$$
\Sigma_{a}(E)=\left\{\sqrt{2(E-\lambda)}: \lambda \in \Lambda_{a}(\lambda \leqq 0)\right\}
$$

for fixed energy $E>0$. By definition, $\sigma \geqq \sqrt{2 E}$ for $\sigma \in \Sigma_{a}(E)$.

Let $S$ be again the unit sphere in $X$. For given cluster decomposition $a$, we define the subset $S_{a}$ in $S$ by

$$
S_{a}=\left\{\omega \in S: \omega_{j}=\omega_{k} \text { if } j a k, \omega_{j} \neq \omega_{k} \text { if } \sim j a k\right\} .
$$

If $\#(a)=2$, then the set $S_{a}$ defined above coincides with the set defined by (1.2). Let $\omega \in S$. Then we can determine a cluster decomposition $a$ uniquely so that $\omega \in S_{a}$. Hence the unit sphere $S$ is decomposed into the disjoint sum of $S_{a}$. We can easily see that if $\omega \in S_{a}$ for some $a$, then all points in a small neighborhood of $\omega$ on $S$ lie in $S_{b}$ with $b \subset a$.

2.2. We are now in a position to formulate the non-propagation estimate in question. The statement is fairly lengthy.

Proposition 2.1. Let energy $E>0$ and $\omega \in S$ be fixed. Assume $\omega$ to be in $S_{b}$ for some cluster decomposition $b$. Then one has the follow'ing statements:

(i) Let $\gamma$ be defined by (1.3). .1ssume that:

(a) $\sigma>0$ is not in $\Sigma_{b}(E)$.

(b) $f \in C_{0}^{\infty}\left(\boldsymbol{R}^{1}\right)$ has support in a small open interval $I I$ of $\sigma$ avoiding all the points in $\Sigma_{b}(E)$.

(c) $Q(x) \in S^{0}(X)$ has support in

$$
\text { Cone }(\omega, \delta)=\{r \in X:|r|>1,|r /| r|-\omega|<\delta\} .
$$

Then one can take $\delta, 0<\delta \ll 1$, interval $\Pi$ around $\sigma$ and interval $\Gamma$ around E so small that

$$
\int\left\|\langle x\rangle^{-1 / 2} Q(x) f(\gamma) \exp (-i t H) E_{\Gamma}(H) \psi\right\|_{X}^{2} d t \leqq C\|\phi\|_{X}^{2},
$$

where $E_{\Gamma}(H)$ denotes the spectral resolution of $H$ onto $\Gamma$.

(ii) Let $\beta, 0<\beta \ll 1$, and $\sigma>0$ be fixed. Let $Q(x)$ be as above. Assume that: (a) $f \in C_{0}^{\infty}\left(\boldsymbol{R}^{1}\right)$ has support in a small open interval $I I$ of $\sigma$.

(b) $F \in S^{0}\left(Z_{b}^{\prime}\right)$ has support in $\left\{q_{b} \in Z_{b}^{\prime}:\left|q_{b}-\sigma \omega\right|>\beta\right\}, \omega \in S_{b}$ being identified with a point in $Z_{b}^{\prime}$. 
Then one can take $\delta, 0<\delta \ll 1$, interval $\Pi$ around $\sigma$ and interval $\Gamma$ around E so small that

$$
\int\left\|\langle x\rangle^{-1 / 2} Q(x) F\left(D_{b}\right) f(\gamma) \exp (-i t H) E_{\Gamma}(H) \psi\right\|_{X}^{2} d t \leqq C\|\boldsymbol{\phi}\|_{X}^{2} .
$$

(iii) The same estimates as in (i) and (ii) remain true for the truncated Hamiltonians $H_{a}$.

Remark. We may say that the operators $\langle x\rangle^{-1 / 2} Q(x) f(\gamma)$ and $\langle x\rangle^{-1 / 2} Q(x)$ $\times F\left(D_{b}\right) f(\gamma)$ as in the proposition are $H$-smooth (or $H_{a}$-smooth) on $\Gamma$.

The statements (i) and (ii) follow as a special case from Propositions 6.7 and 6.11 of [3], respectively.

2.3. We end this section by making a brief comment on the above proposition. As stated above, the non-propagation estimate has been obtained by [3] for general $N$-body systems, including the case of long-range interactions. However, this estimate has been proved under the additional assumptions that:

$$
\begin{aligned}
& \left.\langle y\rangle^{\theta}\left(\nabla_{y} V_{j k}\right)(-\Delta+1)^{-1}, \theta\right\rangle 1 \text {, is bounded on } L^{2}\left(\boldsymbol{R}_{y}^{3}\right) . \\
& \left(y \cdot \nabla_{y}\right)^{2} V_{j k}(-\Delta+1)^{-1} \text { is bounded on } L^{2}\left(\boldsymbol{R}_{y}^{3}\right) .
\end{aligned}
$$

The above restrictions have been essentially used to guarantee that the limiting absorption principle holds $\left.([11]):\langle x\rangle^{-\beta}(H-(\lambda \pm i \kappa))^{-1}\langle x\rangle^{-\beta}, \beta\right\rangle 1 / 2: L^{2}(X) \longrightarrow$ $L^{2}(X)$ is bounded uniformly in $\kappa, 0<\kappa \ll 1$, and $\lambda \in \Gamma, \Gamma$ being an interval avoiding all threshold and bound state energies of $H$. By the smoothness theorem ([9]), this implies immediately that the multiplication operator $\left.\langle x\rangle^{-\beta}, \beta\right\rangle 1 / 2$, is $H$-smooth on $\Gamma$;

$$
\int\left\|\langle x\rangle^{-\beta} \exp (-i t H) E_{\Gamma}(H) \phi\right\|_{X}^{2} d t \leqq C\|\boldsymbol{\phi}\|_{X}^{2} .
$$

Recently, this principle has been improved by [1] and [14] to remain true only under assumption (V) in the case of short-range interactions. Thus we should note that the non-propagation estimate as in Proposition 2.1 is true without (2.5) and (2.6).

\section{§3. Commutator Calculus}

In this section we make a brief review of the commutator calculus developed by [3] which is used as a basic tool to prove the main lemma. For details, see [3] (Section 5) or [5] (Section 2). 
3.1. We begin by introducing several new notations. Let $B: L^{2}(X) \rightarrow L^{2}(X)$ be a bounded operator. We denote by $\|B\|$ the operator norm of $B$. Let $X_{\beta}$ : $\phi(x) \rightarrow\langle x\rangle^{\beta} \phi(x)$ be the multiplication operator by $\langle x\rangle^{\beta}$. For given operator $B$ (not necessarily bounded) on $L^{2}(X)$, we say that $B$ is of class $O\left(\langle x\rangle^{m}\right)$, if $X_{-m+k} B X_{-k}$ extends to a bounded operator on $L^{2}(X)$ for any real $k$. We also write $B_{1}=B_{2}+O\left(\langle x\rangle^{m}\right)$, if the difference $B_{1}-B_{2}$ is of class $O\left(\langle x\rangle^{m}\right)$.

3.2. Lemma 3.1 (Proposition $5.1([3])$ ). Let $\gamma$ be defined by (1.3). Assume that: (a) $f \in C_{0}^{\infty}\left(\boldsymbol{R}^{1}\right)$; (b) $g \in C_{0}^{\infty}\left(\boldsymbol{R}^{1}\right)$; (c) $F \in S^{0}\left(Z_{a}^{\prime}\right), S^{0}$ being defined by (2.1). Then one has the following statements: (i) $f(\gamma) \in O\left(\langle x\rangle^{0}\right)$; (ii) $g(H) \in O\left(\langle x\rangle^{0}\right)$; (iii) $F\left(D_{a}\right) \in O\left(\langle x\rangle^{0}\right)$, where $F\left(D_{a}\right)$ is defined by (2.2) and is considered as an operator acting on $L^{2}(X)$ as well as on $L^{2}\left(Z_{a}\right)$.

Lemma 3.2 (Proposition $5.1([3]))$. Let $f$ and $g$ be as in Lemma 3.1. Assume that $Q(x) \in S^{m}(X)$. Then:

(i ) $[f(\gamma), g(H)] \in O\left(\langle x\rangle^{-1}\right)$.

(ii) $[Q(x), f(\gamma)] \in O\left(\langle x\rangle^{n-1}\right)$.

(iii) $[Q(x), g(H)] \in O\left(\langle x\rangle^{m-1}\right)$.

Remark. The truncated Hamiltonian $H_{a}$ has the same properties as in the lemmas above.

Lemma 3.3. Let $f, g$ and $Q$ be as in Lemmas 3.1 and 3.2. Assume that $F \in S^{0}\left(Z_{a}^{\prime}\right)$. Then :

(i) $\left[Q(x), F\left(D_{a}\right)\right] \in O\left(\langle x\rangle^{m-1}\right)$.

(ii) $\left[f(\gamma), F\left(D_{a}\right) g\left(H_{a}\right)\right] \in O\left(\langle x\rangle^{-1}\right)$.

Proof. Statement (i) follows again from Proposition 5.1 ([3]). We shall sketch a proof of statement (ii) only.

Define the operator $\langle D\rangle$ acting on $L^{2}(X)$ by

$$
\langle D\rangle u=(2 \pi)^{-9 / 2} \int \exp (i x \cdot \xi)\langle\xi\rangle \hat{u}(\xi) d \xi
$$

and write

$$
F\left(D_{a}\right) g\left(H_{a}\right)=F\left(D_{a}\right)\langle D\rangle^{-1}\langle D\rangle g\left(H_{a}\right) .
$$

Then the commutator in statement (ii) of the lemma is written as

$$
\left[f(\gamma), F\left(D_{a}\right)\langle D\rangle^{-1}\right]\langle D\rangle g\left(H_{a}\right)+F\left(D_{a}\right)\langle D\rangle^{-1}\left[f(\gamma),\langle D\rangle g\left(H_{a}\right)\right] .
$$

By Proposition $5.1([3]),\left[f(\gamma), F\left(D_{a}\right)\langle D\rangle^{-1}\right] \in O\left(\langle x\rangle^{-1}\right)$ and also it is easy to see that $\langle D\rangle g\left(H_{a}\right) \in O\left(\langle x\rangle^{0}\right)$. Thus the first term on (3.1) is of class $O\left(\langle x\rangle^{-1}\right)$

We shall show that the second term is also of class $O\left(\langle x\rangle^{-1}\right)$. To see 
this, we write $\left[f(\gamma),\langle D\rangle g\left(H_{a}\right)\right]$ as

$$
\left[f(\gamma),\langle D\rangle\left(H_{a}+i\right)^{-1}\right]\left(H_{a}+i\right) g\left(H_{a}\right)+\langle D\rangle\left(H_{a}+i\right)^{-1}\left[f(\gamma),\left(H_{a}+i\right) g\left(H_{a}\right)\right] .
$$

The last term is of class $O\left(\langle x\rangle^{-1}\right)$. Since the commutator $\left[\gamma,\langle D\rangle\left(H_{a}+i\right)^{-1}\right]$ is written as

$$
[\gamma,\langle D\rangle]\left(H_{a}+i\right)^{-1}+\langle D\rangle\left(H_{a}+i\right)^{-1}\left[H_{a}, \gamma\right]\left(H_{a}+i\right)^{-1},
$$

it follows from assumption $(\mathrm{V})$ that this commutator is of class $O\left(\langle x\rangle^{-1}\right)$ and hence we have by Lemma 5.3 ([3]) that

$$
\left[f(\gamma),\langle D\rangle\left(H_{a}+i\right)^{-1}\right] \in O\left(\langle x\rangle^{-1}\right) .
$$

This proves that the second term on (3.1) is of class $O\left(\langle x\rangle^{-1}\right)$ and the proof is complete.

3.3. Lemma 3.4. Let $g \in C_{0}^{\infty}\left(\boldsymbol{R}^{1}\right), Q(x) \in S^{0}(X)$ and $\omega \in S$. Assume that: (a) $\omega \in S_{b}$ for some cluster decomposition $b$; (b) $Q$ has support in Cone $(\omega, \delta)$ for $\delta>0$ small enough, Cone $(\omega, \delta)$ being defined by (2.4). If $b$ is a refinement of a cluster decomposition $a$, then

$$
Q(x)\left(g\left(H_{a}\right)-g\left(H_{b}\right)\right) \in O\left(\langle x\rangle^{-\rho}\right)
$$

for $\rho$ as in $(\mathrm{V})$.

Proof. The lemma is easy to prove. By assumption (V), $H_{a}-H_{b}=O\left(|x|^{-\rho}\right)$ as $|x| \rightarrow \infty$ on the support of $Q$. This implies the lemma at once.

\section{$\S 4$. Proof of the Main Lemma}

We are now in a position to prove Lemma 1.1. The proof is very long and is divided into several steps.

Proof of Lemma 1.1. In many situations of the proof, we use without further references the basic fact that the multiplication operator $X_{-\beta}, \beta>1 / 2$, is $H$-smooth or $H_{a}$-smooth on interval $\Gamma$ avoiding the threshold and bound state energies of $H$.

We begin by recalling the notations in Section 1 . Let $E>0$ be fixed. Let $\Gamma$ be a small open interval around $E$, so that $\Gamma$ avoids all the threshold and bound state energies of $H$. Let $g_{0}$ and $g_{1} \in C_{0}^{\infty}(\Gamma)$ be non-negative functions satisfying the relation $g_{1} g_{0}=g_{0}$.

4.1. Lemma 4.1. Let $\left\{j_{a}(x)\right\}_{\#(a)=2}$ be the partition of unity on $X$ as in Section 1. Assume that: (a) $f \in C_{0}^{\infty}\left(\boldsymbol{R}^{1}\right)$; (b) $\omega \in S_{b}$ for some cluster decomposition $b$; (c) $F \in S^{0}\left(Z_{b}^{\prime}\right)$; (d) $Q(x) \in S^{0}(X)$ has support in Cone $(\omega, \delta)$ for some $\delta>0$ small enough. If $b$ is a refinement of a two-cluster decomposition $a$, then one has 


$$
\begin{aligned}
& g_{1}\left(H_{a}\right) f(\gamma)^{2} F\left(D_{b}\right)^{2} Q(x)^{2}\left[H_{0}, j_{a}\right] g_{0}(H) \\
& \quad=f(\gamma) F\left(D_{b}\right) Q(x) X_{-1 / 2} B X_{-1 / 2} Q(x) F\left(D_{b}\right) f(\gamma)+O\left(\langle x\rangle^{-2}\right)
\end{aligned}
$$

with the bounded operator

$$
B=X_{1 / 2} g_{1}\left(H_{b}\right)\left[H_{0}, j_{a}\right] g_{0}\left(H_{b}\right) X_{1 / 2}: L^{2}(X) \longrightarrow L^{2}(X) .
$$

Proof. If we take account of the fact that $F\left(D_{b}\right)$ and $g\left(H_{b}\right)$ commute with each other, the lemma is proved by making repeated use of the commutator calculus in Section 3.

4.2. Let $\omega \in S$ be fixed. Assume $\omega$ to be in $S_{b}$ for some cluster decomposition $b$. Let $\Sigma_{b}(E)$ be defined by (2.3). Recall the representation (1.4) for the term $\phi_{3 a}(t)$ under consideration. Then, by Proposition 2.1 and Lemma 4.1, we see that it suffices to prove the lemma for a term $\eta_{a}(t ; \omega)$ of the form

$$
\eta_{a}=\int_{0}^{\iota} g_{1}\left(H_{a}\right) \exp \left(i s H_{a}\right) f(\gamma) F\left(D_{b}\right) Q(x ; \omega)\left[H_{0}, j_{a}\right] \exp (-i s H) g_{0}(H) \psi d s,
$$

where non-negative functions $Q, f$ and $F$ have the following properties: $(Q)$ $Q \in S^{0}(X)$ has support in Cone $(\omega, \delta)$ for some $\delta>0$ small enough; $(f) f \in C_{0}^{\infty}\left(\boldsymbol{R}^{1}\right)$ has support in a small neighborhood of $\sigma, \sigma \geqq \sqrt{2 E}$, for some $\sigma$ in $\Sigma_{b}(E) ;(F)$ $F \in S^{0}\left(Z_{b}^{\prime}\right)$ has support in $\left\{q_{b} \in Z_{b}^{\prime}:\left|q_{b}-\sigma \omega\right|<\beta\right\}$ for some $\beta>0$ small enough, $\sigma$ being the same as in $(f)$.

Here we should note that the supports of $\nabla_{x} j_{a}$ and $Q(x ; \omega)$ intersect with each other only for a two-cluster decomposition $a$ such that $b$ is a strict refinement of $a ; b \subset a$ and $b \neq a$. Thus we may assume that \#(b) takes the values 3 or 4 only. If, in particular, $\#(b)=4$, then we may also assume that $x /|x| \in S_{b}$ for all $x$ in the support of $Q$, because $S_{b}$ with $\#(b)=4$ is an open set in $S$.

4.3. We now introduce a non-negative smooth function $\chi_{R} \in S^{0}(X), R \gg 1$, with the following properties: (i) $\chi_{R}$ has support in $\{x \in X:|x|>R\}$ and $\chi_{R}=1$ for $|x|>2 R$; (ii) $\left|\partial_{x}^{\alpha} \chi_{R}\right| \leqq C_{\alpha}\langle x\rangle^{-|\alpha|}$ for $C_{\alpha}$ independent of $R$. Define $Q_{R}(x ; \omega)$ as $Q_{R}=\chi_{R} Q(x ; \omega)$ and $\eta_{a R}(t ; \omega)$ as

$$
\eta_{a R}=\int_{0}^{t} g_{1}\left(H_{a}\right) \exp \left(i s H_{a}\right) F\left(D_{b}\right) Q_{R}(x ; \omega) f(\gamma)\left[H_{0}, j_{a}\right] \exp (-i s H) g_{0}(H) \psi d s .
$$

Then the difference $\eta_{a}(t ; \omega)-\eta_{a R}(t ; \omega)$ is strongly convergent in $L^{2}(X)$ as $t \rightarrow \infty$. Therefore, to prove the lemma, it suffices to show that

$$
\limsup _{t \rightarrow \infty}\left\|\sum_{\#(a)=2} \exp \left(-i t H_{a}\right) \eta_{a R}(t ; \omega)\right\|_{X}=o(1) \text { as } R \longrightarrow \infty \text {. }
$$

4.4. By the construction of $\left\{j_{a}(x)\right\}_{\#(a)=2}$,

$$
\sum_{\#(a)=2}\left[H_{0}, j_{a}\right]=0
$$


and hence it follows that

$$
\sum_{\#(a)=2} \exp \left(-i t H_{a}\right) \eta_{a R}(t ; \omega)=\sum_{\#(a)=2} \exp \left(-i t H_{a}\right) \zeta_{a R}(t ; \omega),
$$

where

$$
\begin{aligned}
\zeta_{a R}=\int_{0}^{t} & \left\{g_{1}\left(H_{a}\right) \exp \left(i s H_{a}\right)-\exp \left(i t H_{a}\right) g_{1}\left(H_{b}\right) \exp \left(-i(t-s) H_{b}\right)\right\} \\
& \times F\left(D_{b}\right) Q_{R}(x ; \omega) f(\gamma)\left[H_{0}, j_{a}\right] \exp (-i s H) g_{0}(H) \psi d s .
\end{aligned}
$$

If $b$ is not a strict refinement of $a$, then $Q_{R}(x ; \omega)$ vanishes on the support of $\nabla_{x} j_{a}$, as remarked above, and hence we can easily prove that for such a twocluster decomposition $a$,

$$
\left\|\zeta_{a R}(t ; \omega)\right\|_{X}=o(1), \quad R \longrightarrow \infty,
$$

uniformly in $t \geqq 0$. Thus we have only to consider the term $\zeta_{a R}(t ; \omega)$ with twocluster decomposition $a$ such that $b$ is a strict refinement of $a$. Recall the property (j.3) in Section 1 that $j_{a}(x)$ vanishes on $Y_{a}$ for $|x|>1$. Hence, we may further assume that

$$
Q_{R}(x ; \omega) \quad \text { vanishes on } Y_{a}
$$

for two-cluster decomposition $a$ as above.

We now fix $\mu$ close enough to $1 / 2$ as follows:

$$
\max (0,3 / 2-\rho)<\mu<1 / 2 \text {. }
$$

Then we have

$$
\int_{0}^{\infty}\left\|\chi_{R} X_{\mu} f(\gamma)\left[H_{0}, j_{a}\right] \exp (-i s H) g_{0}(H) \psi\right\|_{X}^{2} d s=o(1)\|\phi\|_{X}^{2}
$$

as $R \rightarrow \infty$. Therefore, (4.1) follows immediately, if it can be proved that

$$
\int_{0}^{t}\left\|\theta_{a R}(t, s ; \omega)\right\|_{X}^{2} d s \leqq C\|\phi\|_{X}^{2}
$$

uniformly in $t \geqq 0$ and $R \gg 1$, where $\theta_{a R}(t, s ; \omega), 0 \leqq s \leqq t$, is defined by

$$
\theta_{a R}=X_{-\mu} Q_{R} F\left(D_{b}\right)\left\{\exp \left(-i s H_{a}\right) g_{1}\left(H_{a}\right)-\exp \left(i(t-s) H_{b}\right) g_{1}\left(H_{b}\right) \exp \left(-i t H_{a}\right)\right\} \phi \text {. }
$$

In the following two sections, we will prove (4.4) separatedly according as $\#(b)=3$ or $\#(b)=4$.

\section{§ 5. Four-Cluster Case}

In this section we prove (4.4) in the case that $b$ is the four-cluster decomposition $b=\{1,2,3,4\}$. For notational brevity, we fix a two-cluster decomposition $a$ as follows :

$$
a=\{1,(2,3,4)\},
$$


so that $b$ is a strict refinement of $a$. By (0.5), the truncated Hamiltonian $H_{a}$ takes the form

$$
H_{a}=K_{a} \otimes I d+I d \otimes T_{a} \quad \text { on } \quad L^{2}(X)=L^{2}\left(Y_{a}\right) \otimes L^{2}\left(Z_{a}\right)
$$

and also we have

$$
H_{b}=H_{0}=K_{0} \otimes I d+I d \otimes T_{a},
$$

where $K_{0}=-(1 / 2) \Delta$ is the free Hamiltonian on $L^{2}\left(Y_{a}\right)$.

5.1. We introduce the coordinates $z_{a}$ on $Z_{a}$ by

$$
z_{a}=\sqrt{3 / 4}\left\{r_{1}-\left(r_{2}+r_{3}+r_{4}\right) / 3\right\}
$$

with the normalization constant $\sqrt{ } 3 / 4$ and we denote by $q_{a} \in Z_{a}^{\prime}$ the coordinates dual to $z_{a}$. As is easily seen, $z_{a}=0$ on $Y_{a}$ only. By (4.2), $\omega$ does not lie on $Y_{a}$ and hence the projection of $\omega$ onto $Z_{a}^{\prime}$ never vanishes. Thus we can find a non-negative symbol $F_{0} \in S^{0}\left(Z_{a}^{\prime}\right)$ such that $F_{0}$ has support in $\left\{q_{a} \in Z_{a}^{\prime}: 1 / d<\right.$ $\left.\left|q_{a}\right|<d\right\}$ for some $d>1$ and that $F\left(q_{b}\right)=F\left(q_{b}\right) F_{0}\left(q_{a}\right)$. By construction, we have

$$
Q_{R}(x ; \omega) F\left(D_{b}\right)=Q_{R}(. x ; \omega) F\left(D_{b}\right) F_{0}\left(D_{a}\right) .
$$

Lemma 5.1. Let $F_{0}$ be as above. Then, for any $\nu>1 / 2$, one has

$$
\int\left\|\left\langle z_{a}\right\rangle^{-\nu} F_{0}\left(D_{a}\right) \exp \left(-i t H_{a}\right) \phi\right\|_{Y}^{2} d t \leqq C\|\boldsymbol{\phi}\|_{Y}^{2}
$$

Proof. By commutativity, we can write

$$
\left\langle z_{a}\right\rangle^{-\nu} F_{0}\left(D_{a}\right) \exp \left(-i t H_{a}\right)=\exp \left(-i t K_{a}\right)\left\langle z_{a}\right\rangle^{-\nu} \exp \left(-i t T_{a}\right) F_{0}\left(D_{a}\right) .
$$

Since the multiplication operator $\left.\left\langle z_{a}\right\rangle^{-\nu}, \nu\right\rangle 1 / 2$, is $T_{a}$-smooth, the lemma follows at once.

By making use of an argument similar to that in the proof of Lemma 5.1, we see that it suffices to prove (4.4) for the term $\sigma_{a R}(t, s ; \omega), 0 \leqq s \leqq t$, defined by

$$
\sigma_{a R}=L\left\{\exp \left(-i s H_{a}\right)-\exp \left(i(t-s) H_{0}\right) \exp \left(-i t H_{a}\right)\right\} F_{0}\left(D_{a}\right) \psi
$$

with $L=Q_{R} F\left(D_{b}\right) g_{1}\left(H_{0}\right)\left\langle z_{a}\right\rangle^{-\mu}$.

5.2. We calculate the difference

$$
\exp \left(-i s K_{a}\right)-\exp \left(i(t-s) K_{0}\right) \exp \left(-i t K_{a}\right) .
$$

Taking into account the relation

$$
K_{a}-K_{0}=\Sigma_{2 \leqq j<k \leqq 4} V_{j k}\left(r_{j}-r_{k}\right),
$$

we represent this difference in the integral form; 


$$
\Sigma_{2 \leqq j<k \leqq 4} i \int_{0}^{t} \exp \left(i(\tau-s) K_{0}\right) V_{j k} \exp \left(-i \tau K_{a}\right) d \tau
$$

and hence we have

$$
\begin{aligned}
& \left\langle z_{a}\right\rangle^{-\mu}\left\{\exp \left(-i s H_{a}\right)-\exp \left(i(t-s) H_{0}\right) \exp \left(-i t H_{a}\right)\right\} F_{0}\left(D_{a}\right) \\
& =\Sigma_{2 \leq j<k \leqq 4} i \int_{s}^{t} \exp \left(i(\tau-s) K_{0}\right) V_{j k} \exp \left(-i \tau K_{a}\right) d \tau\left\langle z_{a}\right\rangle^{-\mu} \exp \left(-i s T_{a}\right) F_{0}\left(D_{a}\right) .
\end{aligned}
$$

We analyze the operator $\exp \left(i \tau K_{0}\right) V_{j k}, \tau \geqq 0$. For brevity, we consider only the operator with the pair $(2,3)$. To do this, we introduce the coordinates $y_{a}=\left(y_{a}^{1}, y_{a}^{2}\right)$ on $Y_{a}$ by

$$
y_{a}^{1}=\sqrt{1 / 2}\left(r_{2}-r_{3}\right), \quad y_{a}^{2}=\sqrt{2 / 3}\left\{r_{4}-\left(r_{2}+r_{3}\right) / 2\right\}
$$

and denote by $p_{a}=\left(p_{a}^{1}, p_{a}^{2}\right) \in Y_{a}^{\prime}$ the coordinates dual to $Y_{a}$. In the above coordinate system, the potential $V_{23}$ is represented in terms of the coordinates $y_{a}^{1}$ only;

and also we have

$$
V_{23}\left(r_{2}-r_{3}\right)=V_{23}\left(\sqrt{2} y_{a}^{1}\right)
$$

$$
K_{0}=K_{01}+K_{02} \text {, }
$$

where $K_{0 j}=-(1 / 2) \Delta, 1 \leqq j \leqq 2$, is represented in terms of the coordinates $y_{a}^{j}$. As is easily seen, the coordinates $y_{a}^{1}$ never vanish on the support of $Q_{R}$ and hence we can find a non-negative symbol $F_{1} \in S^{0}\left(\boldsymbol{R}^{6} \times \boldsymbol{R}^{3}\right)$ such that $F_{1}$ has support in

$$
\left\{\left(y_{a}^{1}, z_{a}, p_{a}^{1}\right): 1 / d<\left|y_{a}^{1}\right| /\left\langle z_{a}\right\rangle,\left|p_{a}^{1}\right|, y_{a}^{1} \cdot p_{a}^{1} /\left|y_{a}^{1}\right|<d\right\}
$$

for some $d>1$ and that

$$
Q_{R}(x ; \omega) F\left(q_{b}\right)=Q_{R}(x ; \omega) F\left(q_{b}\right) F_{1}\left(y_{a}^{1}, z_{a}, p_{a}^{1}\right) 。
$$

With the above symbol $F_{1}$, we associate the pseudodifferential operator

$$
F_{1}\left(y_{a}^{1}, z_{a}, D_{y}^{1}\right) u=(2 \pi)^{-3 / 2} \int \exp \left(i y_{a}^{1} \cdot p_{a}^{1}\right) F_{1}\left(y_{a}^{1}, z_{a}, p_{a}^{1}\right) \hat{u}\left(p_{a}\right) d p_{a}^{1},
$$

$z_{a}$ being regarded as parameters. By relation (5.2), the standard calculus of pseudodifferential operators yields that

$$
Q_{R} F\left(D_{b}\right) g_{1}\left(H_{0}\right)=g_{1}\left(H_{0}\right) Q_{R} F\left(D_{b}\right) F_{1}\left(y_{a}^{1}, z_{a}, D_{y}^{1}\right)+O\left(\langle x\rangle^{-1}\right) .
$$

Lemma 5.2. Let $K_{01}=-(1 / 2) \Delta$ be again the free Hamiltonian in the coordinates $y_{a}^{1} \in \boldsymbol{R}^{3}$. Let $F_{1}$ be as above. Then, for any $k \geqq 0$, one has

$$
\left\|\left\langle y_{a}^{1}\right\rangle^{-k} \exp \left(-i \tau K_{01}\right) F_{1}\left(y_{a}^{1}, z_{a}, D_{y}^{1}\right)^{*}\right\| \leqq C\left(1+\tau+\mid z_{a} !\right)^{-k}, \tau \geqq 0,
$$

where $\|\cdot\|$ denotes the uniform operator norm when considered as an operator acting on the $L^{2}$ space over the $y_{a}^{1}$-space. 
Proof. The lemma is easy to prove. We give only a sketch for the proof. We write explicitly the term on the left side of the inequality in the lemma by use of the Fourier transform. If we take account of the fact that the outgoing free particle with initial state $\left(y_{a}^{1}, p_{a}^{1}\right)$ in the support of $F_{1}, z_{a}$ being regarded as parameters, is in the region $\left\{y_{a}^{1}:\left|y_{a}^{1}\right|>C\left(1+\tau+\left|z_{a}\right|\right)\right\}$ at time $\tau \geqq 0$, the lemma is proved by making repeated use of partial integration.

5.3. We now complete the proof of (4.4). Recall the representation for the term $\sigma_{a R}(t, s ; \omega)$. By (5.4), Lemma 5.2 with $k=\rho, \rho$ being as in $(\mathrm{V})$, implies that

$$
\left\|\sigma_{a R}(t, s ; \omega)\right\|_{X} \leqq C\left\|\left\langle z_{a}\right\rangle^{-\nu} \exp \left(-i s T_{a}\right) F_{0}\left(D_{a}\right) \psi\right\|_{X}
$$

with $\nu=\rho+\mu-1-\varepsilon>1 / 2, \varepsilon>0$ being fixed small enough. This, together with Lemma 5.1 , proves $(4.4)$ for the case $\#(b)=4$.

\section{$\S 6$. Three-Cluster Case}

The present section is devoted to proving (4.4) for the remaining case $F(b)=3$. The proof is done by making use of the same argument as in the previous case. For notational brevity, we take the three-cluster decomposition $b$ as

$$
b=\{1,2,(3,4)\}
$$

and fix a two-cluster decomposition $a$ again as

$$
a=\{1,(2,3,4)\}
$$

so that $b$ is a strict refinement of $a$.

In the above situation, the truncated Hamiltonians $H_{a}$ and $H_{b}$ take the forms

$$
\begin{aligned}
& H_{a}=K_{a} \otimes I d+I d \otimes T_{a}, \\
& H_{b}=K_{b} \otimes I d+I d \otimes T_{a}
\end{aligned}
$$

on $L^{2}(X)=L^{2}\left(Y_{a}\right) \otimes L^{2}\left(Z_{a}\right)$, where $T_{a}=-(1 / 2) \Delta$ is represented in terms of the coordinates $z_{a}$ defined by (5.1).

6.1. We first look at the difference $K_{a}-K_{b}$. To this end, we introduce the coordinates $y_{a}=\left(y_{a}^{1}, y_{a}^{2}\right)$ on $Y_{a}$ by

$$
y_{a}^{1}=\sqrt{1 / 2}\left(r_{3}-r_{4}\right), \quad y_{a}^{2}=\sqrt{2 / 3}\left\{r_{2}-\left(r_{3}+r_{4}\right) / 2\right\},
$$

so that

$$
r_{2}-r_{3}=\sqrt{3 / 2} y_{a}^{2}-\sqrt{1 / 2} y_{a}^{1}, \quad r_{2}-r_{4}=\sqrt{3 / 2} y_{a}^{2}+\sqrt{1 / 2} y_{a}^{1} .
$$

In the above coordinate system, the Hamiltonian $K_{b}$ acting on $L^{2}\left(Y_{a}\right)$ has the representation 


$$
K_{b}=K_{1}+K_{02},
$$

where $K_{02}=-(1 / 2) \Delta$ is represented in terms of the coordinates $y_{a}^{2}$ and also the two-body subsystem Hamiltonian

$$
K_{1}=-\frac{1}{2} \Delta+V_{34}\left(\sqrt{2} y_{a}^{1}\right)
$$

is represented in terms of the coordinates $y_{a}^{1}$ only. Thus the difference in question is written as

$$
K_{a}-K_{b}=V_{23}\left(r_{2}-r_{3}\right)+V_{24}\left(r_{2}-r_{4}\right)
$$

with the coordinates $r_{2}-r_{3}$ and $r_{2}-r_{4}$ represented in terms of $y_{a}$ through relation (6.2).

6.2. Next we analyze the operator $Q_{R}(x ; \omega) F\left(D_{b}\right) g_{1}\left(H_{b}\right)$. Let $q_{a} \in Z_{a}^{\prime}$ be again the coordinates dual to $z_{a}$. We denote by $p_{a}=\left(p_{a}^{1}, p_{a}^{2}\right) \in Y_{a}^{\prime}$ the coordinates dual to $y_{a}$ defined by (6.1). Then we can take $q_{b}=\left(p_{a}^{2}, q_{a}\right)$ as a coordinate system on $Z_{b}^{\prime}$. If $\omega \in S_{b}$ with $b=\{1,2,(3,4)\}$ and if we represent $\omega$ in terms of the coordinates $q_{b}, \omega$ being identified with an element in $Z_{b}^{\prime}$, then the $p_{a}^{2}$-component of $\omega$ never vanishes and it follows from (4.2) that $q_{a}$-component also never vanishes. Recall the property $(F)$ in Section 4 that $F \in S^{0}\left(Z_{b}^{\prime}\right)$ is supported in a small neighborhood of $\sigma \omega$ for $\sigma, \sigma \geqq \sqrt{2 E}$, in $\Sigma_{b}(E)$. Hence there exists $d>1$ such that

$$
\operatorname{supp} F \subset\left\{q_{b}=\left(p_{a}^{2}, q_{a}\right): 1 / d<\left|p_{a}^{2}\right|,\left|q_{a}\right|<d\right\} .
$$

Let $K_{1}$ be defined by (6.3). Then we have $H_{b}=-(1 / 2) \Delta+K_{1}$, where $\Delta$ is the Laplace operator in the coordinate system $z_{b}=\left(y_{a}^{2}, z_{a}\right)$. Recall that $g_{1} \in$ $C_{0}^{\infty}(\Gamma)$ has support in a small neighborhood of $E>0$ and that $\left|q_{b}\right|^{2} / 2>E-\delta$ for $\delta>0$ small enough on the support of $F$. Thus we can write the operator $Q_{R} F\left(D_{b}\right) g_{1}\left(H_{b}\right)$ under consideration as follows:

$$
Q_{R} F\left(D_{b}\right) g_{1}\left(H_{b}\right) F_{1}\left(y_{a}^{1}, z_{a}\right) \tilde{g}\left(K_{1}\right) F_{2}\left(y_{a}^{2}, z_{a}, D_{y}^{2}\right) F_{0}\left(D_{a}\right)+O\left(\langle x\rangle^{-1}\right),
$$

where the non-negative functions $F_{j}, 0 \leqq j \leqq 2$, and $\tilde{g}$ have the following properties: $\left(F_{0}\right) \quad F_{0} \in S^{0}\left(\boldsymbol{R}^{3}\right)$ has support in $\left\{q_{a} ; 1 / d_{0}<\left|q_{a}\right|<d_{0}\right\}$ for some $d_{0}>1$; $\left(F_{1}\right) F_{1} \in S^{0}\left(\boldsymbol{R}^{6}\right)$ has support in $\left\{\left(y_{a}^{1}, z_{a}\right) ;\left|y_{a}^{1}\right|\left\langle d_{1}\left\langle z_{a}\right\rangle\right\}\right.$ for some $d_{1}>0$ small enough; $\left(F_{2}\right) F_{2} \in S^{0}\left(\boldsymbol{R}^{6} \times \boldsymbol{R}^{3}\right)$ has support in

$$
\left\{\left(y_{a}^{2}, z_{a}, p_{a}^{2}\right): 1 / d_{2}<\left|y_{a}^{2}\right| /\left\langle z_{a}\right\rangle,\left|p_{a}^{2}\right|, y_{a}^{2} \cdot p_{a}^{2} /\left|y_{a}^{2}\right|<d_{2}\right\}
$$

for some $d_{2}>1$; $(\tilde{g}) \tilde{g} \in C_{0}^{\infty}\left(\boldsymbol{R}^{1}\right)$ has support in $\left(-\infty, d_{1}^{2} / 2\right), d_{1}$ being the same as in $\left(F_{1}\right)$. Here the pseudodifferential operator $F_{2}\left(y_{a}^{2}, z_{a}, D_{y}^{2}\right)$ with symbol $F_{2}\left(y_{a}^{2}, z_{a}, p_{a}^{2}\right)$ is defined as in (5.3) and also we may assume that $d_{1}$ is so small that 


$$
0<d_{1}<\frac{1}{100} d_{2}^{-1}
$$

Lemma 6.1. Let $K_{02}=-(1 / 2) \Delta$ be again the free Hamiltonian in the coordinates $y_{a}^{2}$. Let $F_{2}$ be as above. Denote by $\chi\left(\left|y_{a}^{2}\right|<d\left\langle z_{a}\right\rangle\right)$ the characteristic function of the indicated region in the $y_{a}^{2}$-space. Then one has

$$
\begin{aligned}
\| \chi\left(\left|y_{a}^{2}\right|<\frac{1}{2} d_{2}^{-1}(\tau+\right. & \left.\left.\left\langle z_{a}\right\rangle\right)\right) \exp \left(-i \tau K_{02}\right) F_{2}\left(y_{a}^{2}, z_{a}, D_{y}^{2}\right)^{*} \| \\
& \leqq C_{N}\left(1+\tau+\left|z_{a}\right|\right)^{-N}, \quad \tau \geqq 0,
\end{aligned}
$$

for any $N \gg 1$, where $\|\cdot\|$ denotes the uniform operator norm when considered as an operator acting on the $L^{2}$ space over the $y_{a}^{2}$-space.

Proof. The lemma is proved by the same reasoning as in the proof of Lemma 5.2. We have only to note that the outgoing free particle with initial state $\left(y_{a}^{2}, p_{a}^{2}\right)$ in the support of $F_{2}$ never pass over the region $\left\{y_{a}^{2} ;\left|y_{a}^{2}\right|<\right.$ $\left.(1 / 2) d_{2}^{-1}\left(\tau+\left\langle z_{a}\right\rangle\right)\right\}$ at time $\tau \geqq 0$.

6.4. Lemma 6.2. Let $K_{1}$ be defined by (6.3). Let $F_{1}$ and $\tilde{g}$ be as above. Then, for $\left|z_{a}\right| \gg 1$, one has

$$
\begin{aligned}
\| \chi\left(\left|y_{a}^{1}\right|>\right. & \left.2 d_{1}\left(\tau+\left\langle z_{a}\right\rangle\right)\right) \exp \left(-i \tau K_{1}\right) \tilde{g}\left(K_{1}\right) F_{1}\left(y_{a}^{1}, z_{a}\right) \| \\
& \leqq C\left(1+\tau+\left|z_{a}\right|\right)^{-\rho}, \quad \tau \geqq 0,
\end{aligned}
$$

with $\rho$ as in $(V)$, where $\|\cdot\|$ denotes the uniform operator norm when considered as an operator acting on the $L^{2}$ space over the $y_{a}^{1}$-space.

A similar result has been already proved by Enss [4], although the formulation of the result there takes a slightly different form. For completeness, we will prove the lemma above in the last section.

6.5. We proceed with the argument, accepting Lemma 6.2 as proved. As $n$ the case $\#(b)=4$, we represent the difference

$$
\exp \left(-i s K_{a}\right)-\exp \left(i(t-s) K_{b}\right) \exp \left(-i t K_{a}\right)
$$

in the integral form. By (6.4), this difference is written as

$$
i \int_{s}^{t} \exp \left(i(\tau-s) K_{b}\right)\left\{V_{23}+V_{24}\right\} \exp \left(-i \tau K_{a}\right) d \tau \text {. }
$$

We now recall the relation (6.2). If $\left|y_{a}^{2}\right|>(1 / 2) d_{2}^{-1}\left(|\tau-s|+\left\langle z_{a}\right\rangle\right)$ and $\left|y_{a}^{1}\right|<$ $2 d_{1}\left(|\tau-s|+\left\langle z_{a}\right\rangle\right)$ with $\left(d_{1}, d_{2}\right)$ as in (6.5), then

$$
\left|r_{2}-r_{3}\right| \geqq C\left\langle y_{a}^{2}\right\rangle
$$

and hence $\left|V_{23}\right| \leqq C\left\langle y_{a}^{2}\right\rangle^{-\rho}$ for $\left(y_{a}^{1}, y_{a}^{2}\right)$ as above; similarly for $V_{24}$. Thus, 
Lemmas 5.1, 6.1 and 6.2 enable us to follow the same argument as in the case $\#(b)=4$ and we can prove $(4.4)$ for the remaining case $\#(b)=3$ also. Thus the proof of the main lemma is now complete, although it remains to prove Lemma 6.2 .

\section{$\S 7$. Non-Propagation Property in Two-Body Systems}

In this section we prove Lemma 6.2. As stated in Section 6, a similar result has been already obtained by Enss [4], including the case of long-range interactions. The idea of proof is essentially the same as in [4], although the proof in the case of short-range interactions is much more simplified.

We begin by rewriting the statement of Lemma 6.2 in the more simplified form. Consider the two-body Schrödinger operator

$$
T=-\frac{1}{2} \Delta+v \quad \text { on } \quad L^{2}\left(\boldsymbol{R}_{y}^{3}\right),
$$

where the real potential $v(y), y \in \boldsymbol{R}^{3}$, is assumed to have the following decaying property:

$$
|v(y)| \leqq C(1+|y|)^{-o}, \quad \rho>1 .
$$

Let $g \in C_{0}^{\infty}\left(\boldsymbol{R}^{1}\right)$ be a non-negative function with support in $\left(-\infty, \lambda^{2} / 2\right), \lambda>0$, and denote by $\chi(\cdot)$ the characteristic function of the indicated region in the $y$-space.

Proposition 7.1. Let the notations be as above. Assume (7.1). Denote by $\|\cdot\|$ the uniform operator norm when considered as an operator acting on $L^{2}\left(\boldsymbol{R}_{y}^{3}\right)$. Then one has

$$
\|\chi(|y|>(1+\delta)(R+\lambda t)) \exp (-i t T) g(T) \chi(|y|<R)\| \leqq C_{\tilde{o}}(1+t+R)^{-\rho}
$$

uniformly in $t \geqq 0$ and $R \gg 1$ large enough, where $\delta, 0<\delta \ll 1$, is fixed arbitrarily but small enough.

Proof. The proof is done through a series of steps.

(i) Let $T_{0}=-(1 / 2) \Delta$ be the free Hamiltonian on $L^{2}\left(\boldsymbol{R}_{y}^{3}\right)$. Let $\tilde{g} \in C_{0}^{\infty}\left(\mathbb{R}^{1}\right)$ be a non-negative function with support in $\left(-\infty, \lambda^{2} / 2\right)$ such that $\tilde{g}=1$ on the support of $g$. For notational brevity, we set

Since

$$
F_{t}=\chi(|y|>(1+\delta)(R+\lambda t)), \quad t \geqq 0 \text {. }
$$

$$
\left\|\langle y\rangle^{\circ}\left(\tilde{g}(T)-\tilde{g}\left(T_{0}\right)\right)\right\|<\infty,
$$

it suffices to prove that

$$
\left\|F_{t} \tilde{g}\left(T_{0}\right) \exp (-i t T) g(T) \chi(|y|<R)\right\| \leqq C_{\delta}(1+t+R)^{-\rho}
$$

with another $C_{\delta}$. 
(ii) We can write the term on the left side of (7.3) in the integral foi $m$;

$$
\begin{aligned}
& F_{t} \tilde{g}\left(T_{0}\right) \exp \left(-i t T_{0}\right) g(T) \chi(|y|<R) \\
& \quad-i \int_{0}^{t} F_{t} \tilde{g}\left(T_{0}\right) \exp \left(-i(t-\tau) T_{0}\right) v \exp (-i \tau T) g(T) \chi(|y|<R) d \tau .
\end{aligned}
$$

Define the multiplication operator $B_{t}, t \geqq 0$, by

$$
B_{t}=\chi(|y|<(1+\delta / 2) R+(\delta / 2) \lambda t)
$$

and decompose $g(T)$ as

$$
g(T)=B_{t} g(T)+\left(I d-B_{t}\right) g\left(T_{0}\right)+\left(I d-B_{t}\right)\left(g(T)-g\left(T_{0}\right)\right) .
$$

Lemma 7.2. One has the following estimates:

(a) $\left\|F_{t} \exp \left(-i \tau T_{0}\right) \tilde{g}\left(T_{0}\right) B_{t}\right\| \leqq C_{N}(1+t+R)^{-N}, \quad 0 \leqq \tau \leqq t, \quad$ for $\quad N \gg 1$.

(b) $\left\|\left(I d-B_{t}\right) g\left(T_{0}\right) \chi(|y|<R)\right\| C_{N}(1+t+R)^{-N}$ for $N \gg 1$.

(c) $\left\|\left(I d-B_{t}\right)\left(g(T)-g\left(T_{0}\right)\right)\right\| \leqq(1+t+R)^{-\rho}$.

Proof. (a) The term under consideration can be explicitly represented by making use of the Fourier transform. By definition, the free particle with energy in $\left[0, \lambda^{2} / 2\right)$ starting from the support of $B_{t}$ at time $\tau=0$ never reach the support of $F_{t}$ at time $\tau, 0 \leqq \tau \leqq t$. Hence the standard argument using integration by parts proves (a). (b) This is an immediate consequence of Lemma 2.2 ([4]). (c) This follows from (7.2) at once.

By Lemma 7.2, we see that the first term in (7.4) obeys the desired estimate.

(iii) We study the second term in (7.4). We first decompose the potential $v$ as

where

$$
v=\sum_{j=1}^{3} U_{j}(\tau), \quad 0 \leqq \tau \leqq t,
$$

$$
U_{1}=v F_{\tau} \tilde{g}\left(T_{0}\right), \quad U_{2}=v F_{\tau}\left(I d-\tilde{g}\left(T_{0}\right)\right), \quad U_{3}=v\left(I d-F_{\tau}\right) .
$$

According to the decomposition above, we write the second term in question as $i \sum_{j=1}^{3} L_{j}$, where

$$
L_{j}=\int_{0}^{t} F_{t} \tilde{g}\left(T_{0}\right) \exp \left(-i(t-\tau) T_{0}\right) U_{j}(\tau) \exp (-i \tau T) g(T) \chi(|y|<R) d \tau .
$$

By Lemma 7.2, (a), the first term $L_{1}$ is evaluated as

$$
\left\|L_{1}\right\|=o(1) \sup _{0 \leqq \tau \leqq t}\left\|F_{\tau} \tilde{g}\left(T_{0}\right) \exp (-i \tau T) g(T) \chi(|y|<R)\right\|
$$

as $R \rightarrow \infty$ uniformly in $t \geqq 0$. The second term $L_{2}$ is also easy to evaluate Write

$$
U_{2}(\tau) \exp (-i \tau T) g(T)=\imath F_{\tau}\left(\tilde{g}(T)-\tilde{g}\left(T_{0}\right)\right) \exp (-i \tau T) g(T)
$$


By (7.2), we have

$$
\left\|\left(I d-B_{t}\right) v\left(\tilde{g}(T)-\tilde{g}\left(T_{0}\right)\right)\right\| \leqq C(1+t+R)^{-2 \rho} .
$$

This, again together with Lemma 7.2, (a), implies that

$$
\left\|L_{2}\right\| \leqq C(1+t+R)^{-\rho} \text {. }
$$

Lemma 7.3. For any $N \gg 1$, one has

$$
\left\|F_{t} \tilde{g}\left(T_{0}\right) \exp \left(-i(t-\tau) T_{0}\right)\left(I d-F_{\tau}\right)\right\| \leqq C_{N}(1+|t-\tau|)^{-N}, \quad 0 \leqq \tau \leqq t .
$$

The lemma above is verified in the same way as in the proof of Lemma 7.2 , (a).

Let $U_{3}(\tau)$ be as in (7.6). If we write

$$
U_{3}(\tau)=B_{t} v\left(I d-F_{\tau}\right)+\left(I d-B_{t}\right) v\left(I d-F_{\tau}\right),
$$

it then follows from Lemmas 7.2 and 7.3 that

$$
\left\|L_{3}\right\| \leqq C(1+t+R)^{-\rho} .
$$

Thus, combining (7.7) (7.9) proves the lemma.

\section{References}

[1] Amrein. W., Berthier, A.M. and Georgescue, V., On Mourre's approach to spectral theory, Helv. Phys. Acta., 62 (1989), 1-20.

[2] Cycon, H.L., Froese, R.G., Kirsch, W. and Simon, B.. Schrödinger Operators, Texts and Monographs in Physics, Springer-Verlag, 1987.

[3] Dereziński, J., A new proof of the propagation theorem for $N$-body quantum systems, Commun. Math. Phys., 122 (1989), 203-231,

[4] Enss, V., Propagation properties of quantum scattering states, J. Func. Anal., 52 (1983), 219-251.

[5] - Quantum scattering theory for two- and three-body systems with potentials of short and long range, Schrödinger Operators, Lect. Notes in Math., 1159, Springer-Verlag, 1984.

[6] - Introduction to asymptotic observables for multiparticle quantum scattering, Schrödinger Operators, Lect. Notes in Math., 1218, Springer-Verlag, 1985.

[7] Froese, R.G. and Herbst, I.W., Exponential bounds and absence of positive eigenvalues for $N$-body Schrödinger operators, Commun. Math. Phys., 87 (1982), 429447.

[8] Hagedorn, G., Asymptotic completeness for classes of two, three and four particle Schrödinger operators, Trans. AMS, 258 (1980), 1-75.

[9] Kato, T., Wave operators and similarity for some non-selfadjoint operators, Math. Ann., 162 (1966), 258-279.

[10] Kitada, H., On the completeness of $N$-body wave operators, Preprint, University of Tokyo, 1989.

[11] Perry, P., Sigal, I.M. and Simon, B., Spectral analysis of $N$-body Schrödinger operators, Ann. of Math., 114 (1981), 519-567.

[12] Reed, M. and Simon, B., Methods of Mathematical Physics III, Scattering Theory, Academic Press, 1978. 
[13] Sigal, I. M. and Soffer, A., The $N$-particle scattering problem: Asymptotic completeness for short-range systems, Ann. of Math., 125 (1987), 35-108.

[14] Tamura, H., Principle of limiting absorption for $N$-body Schrödinger operators, -A remark on the commutator method, Lett. in Math. Phys., 17 (1989), 31-36.

[15] - Asymptotic completeness for three-body Schrödinger operators with shortrange interactions, J. Math. Soc. Japan, 43 (1991), 1-24. 
